Mots. Les langages du politique

Regards sur le post-colonialisme linguistique

\title{
Le discours eurorégional. Indices convergents de légitimation d'un espace institutionnel
}

The euroregional discourse. Convergent indications of institutional legitimacy El discurso eurorregional. Indicios convergentes de legitimación institucional

\section{Marie-Hélène Hermand}

\section{OpenEdition}

\section{Journals}

Édition électronique

URL : https://journals.openedition.org/mots/21793

DOI : $10.4000 /$ mots. 21793

ISSN : 1960-6001

Traduction(s) :

The Euroregional discourse. Increasing evidence towards legitimizing an institutional space - URL : https://journals.openedition.org/mots/21839 [en]

\section{Éditeur}

ENS Éditions

\section{Édition imprimée}

Date de publication : 31 décembre 2014

Pagination : 71-86

ISBN : 978-2-84788-544-6

ISSN : 0243-6450

\section{Référence électronique}

Marie-Hélène Hermand, «Le discours eurorégional. Indices convergents de légitimation d'un espace institutionnel », Mots. Les langages du politique [En ligne], 106 | 2014, mis en ligne le 31 décembre 2016, consulté le 07 décembre 2022. URL : http://journals.openedition.org/mots/21793; DOI : https:// doi.org/10.4000/mots. 21793 


\section{Le discours eurorégional. Indices convergents de légitimation d'un espace institutionnel}

Dans le cadre d'une recherche consacrée à une forme méconnue, mais toujours plus visible, de la communication européenne, nous confrontons différents discours dédiés aux eurorégions. Ces «organisations de coopération transfrontalière formées le long des frontières européennes ${ }^{1}$, potentiellement importantes pour l'avenir de l'Europe, fournissent l'occasion de saisir l'expression discrète d'un espace européen qui connaît aujourd'hui de profondes remises en cause.

L'analyse des énoncés institutionnels, à laquelle nous proposons de consacrer cet article, vise à repérer les indices qui concourent à l'élaboration d'un discours présentant les eurorégions comme la configuration incontournable de la future Europe. Elle annonce la correspondance avec les engagements de légitimation de la coopération transfrontalière, exprimés dès 1980 dans la Charte de Madrid $^{2}$ et repris par la Commission européenne dans la présentation préliminaire de son budget 2014-2020 : il s'agit de «renforcer la coopération transfrontalière et [de] faciliter la mise en place d'un plus grand nombre de projets transfrontaliers »3.

Nos premières observations nous amènent à postuler l'existence de trois régularités discursives relatives à l'espace eurorégional, porte d'entrée féconde dans le corpus : les indices d'une légitimation institutionnelle, la systématisation d'objectifs de performance et l'idéalisation d'un cadre de vie offert aux citoyens transfrontaliers. Nous envisageons ici la première de ces régularités, à savoir la dynamique discursive de légitimation de l'espace eurorégional. En amont de l'analyse, notre démarche s'attache à préciser l'inscription du discours dans son environnement institutionnel puis à décrire les conditions

1. Lexique d'aménagement du territoire européen (Université de Paris 7 - Datar - CNRS), http:// www.ums-riate.fr/lexique/modeleterme.php?id=21 (consulté le 5 avril 2014).

2. La Convention-cadre européenne sur la coopération transfrontalière des collectivités ou autorités territoriales, dite Charte de Madrid, a été signée à Madrid le 21 mai 1980 par les États membres du Conseil de l'Europe.

3. Commission européenne, 19 novembre 2013 : http://europa.eu/rapid/press-release_MEMO13-1011_fr.htm, consulté le 7 avril 2014.

Université libre de Bruxelles, Centre de recherche ReSIC marie-helene.hermand@ulb.ac.be 
de la constitution d'un corpus numérique et multilingue, préalables nécessaires à l'examen de la communication dédiée aux zones transfrontalières européennes.

\section{Inscription dans l'histoire de la coopération transfrontalière}

Sous de multiples variantes linguistiques (Euregio, Eurégio, Eurorégion, Europaregion, Regio...), l'eurorégion recouvre des réalités variées, allant de l'association de collectivités territoriales à des organismes de droit privé ou public. Souvent autoproclamées au départ, les eurorégions puisent leurs origines dans les démarches de coopération transfrontalières entreprises à la fin de la seconde guerre mondiale pour maintenir la paix en Europe4. Objet d'étude des géographes, des politologues et des juristes, elles ont fait l'objet d'un récent bornage en science politique :

Les eurorégions peuvent ainsi se définir comme des organisations européennes de coopération transfrontalière et transnationale, plus ou moins structurées, regroupant des autorités territoriales allant en général de la commune à la région ou à leurs équivalents, associées pour la réalisation d'actions et d'objectifs communs, en fonction d'intérêts partagés et dans le cadre de «territoires de projets». (Perrin, 2013, p.7)

Sur 163 régions frontalières dénombrées dans l'Union européenne5, le Conseil de l'Europe recense environ 90 eurorégions qui récoltent les fruits du travail d'influence mené au niveau européen par l'Assemblée des régions frontalières européennes (ARFE) depuis 1971 (Alliès, 2011). Souligné en 1993 dans la Déclaration de Vienne ${ }^{6}$, le rôle des eurorégions pour la stabilité démocratique de l'espace européen est affirmé et défendu par la Commission européenne qui encourage, avec la mise en œuvre du programme Interreg, le financement de projets transfrontaliers. Les années quatre-vingt-dix marquent donc à la fois une étape d'encouragement explicite de la politique de coopération transfrontalière, l'autonomisation de la démarche transfrontalière et la multiplication des eurorégions. En accompagnement du processus d'extension européenne, la politique de voisinage lancée en 2003 par l’Union européenne accentue ensuite cette tendance en élargissant les dispositifs transfrontaliers aux pays d'Europe centrale et orientale.

L’instauration en 2006 du Règlement européen sur le GECT (Groupement européen de coopération territoriale) par le Parlement européen et le Conseil7

4. Le premier projet d’Eurégion voit le jour en 1958 sur la base d'initiatives dispersées entre cinq régions d'Allemagne et des Pays-Bas.

5. http://www.aebr.eu/fr/membres/repartition_regionale.php (consulté le 5 avril 2014).

6. La Déclaration de Vienne, signée le 9 octobre 1993, est disponible sur le site du Conseil de l'Europe.

7. Le Règlement nº $1082 / 2006$ du Parlement européen et du Conseil, du 5 juillet 2006, relatif à 
a déjà permis à 46 eurorégions de se structurer officiellement tandis que 19 autres ont introduit une demande d'enregistrement ${ }^{8}$. D'une portée à la fois symbolique et juridique, le GECT s'inscrit dans les logiques observées de régionalisation en Europe de l'Ouest (Cole, Palmer, 2009) et de l'Est (Wassemberg, 2009). Basé sur la liberté contractuelle des régions membres, il constitue à la fois un instrument de la politique de coopération européenne et un puissant moteur du développement actuel de l'eurorégionalisme (Marin, 2012). Réalité peu familière au grand public, les eurorégions structurées juridiquement concernent pourtant à elles seules plus de 75 millions d'administrés9.

Les principaux acteurs politiques en présence sont la Commission européenne, les régions et les États membres. La méthode communautaire ${ }^{10}$ régit le dialogue entre les protagonistes selon les principes de subsidiarité et de partenariat qui encouragent le financement de projets transfrontaliers et l'invention de nouveaux modèles de collaboration entre les zones frontalières. Ces procédures de soutien sont cependant entravées par des normes nationales jugées contraires aux objectifs européens d'unification des régions frontalières (Alliès, 2011) et, plus globalement, par le degré variable d'engagement des États dans la politique de coopération (Perrin, 2011). Le Règlement sur le GECT, revu en mai 2013, autorise désormais la création de GECT par approbation tacite des États, clarifie la situation juridique de leurs personnels et ouvre des possibilités de partenariats à toutes les entreprises publiques. Le rapport initial de subordination évolue ainsi vers un rapport de concertation et de négociation entre autorités étatiques et infra-étatiques (Goehrs, 2013).

Dotées ou en cours d'acquisition d'un statut, les eurorégions font l'objet d'attentions particulières de l'Union européenne et se présentent aujourd'hui comme le lieu d'expression de nouvelles stratégies de développementterritoria ${ }^{11}$ et de nouvelles configurations de pouvoir. Si elles bénéficient des retombées de l'augmentation des budgets européens dédiés à la cohésion économique et

un groupement européen de coopération territoriale (GECT) est disponible au Journal officiel L 210 du 31 juillet 2006.

8. Le statut s'est installé de manière remarquable depuis 2010:6 nouveaux GECT ont été créés en 2012 et 11 en 2013. La liste des GECT (datée du 4 février 2014) est disponible dans le registre tenu par le Comité des régions : https://portal.cor.europa.eu/egtc/en-US/CoRActivities/Pages/welcome.aspx (consulté le 29 mai 2014).

9. GECT-Rapport de suivi 2013, disponible sur le site du Comité des régions (consulté le 29 mai 2014): http://cor.europa.eu/en/documentation/studies/Documents/EGTC-monitoring-report-2013/ EGTC-monitoring-report-2013-exec-sum-FR.pdf.

10. La méthode communautaire désigne le mode de fonctionnement institutionnel du premier pilier de l’Union européenne, à savoir les communautés européennes. Elle repose sur une logique d'intégration. Les éléments principaux de sa composition sont décrits sur le site de l'Union européenne : http://europa.eu/legislation_summaries/glossary/community_intergovernmental_ methods_fr.htm (consulté le 19 mai 2014).

11. Déclaration commune au 13e sommet de la Grande Région, 24 janvier 2013, p. 7 (consulté le 5 avril 2014) : http://www.granderegion.net/fr/news/2013/01/20130124-13e-sommet-de-lagrande-region/13-SOMMET-DECLARATION-COMMUNE.pdf. 
sociale et de la forte mobilisation des collectivités territoriales ${ }^{12}$, elles doivent désormais être accompagnées d'une communication professionnelle envers les citoyens. Pour ces raisons, nous pensons que la reconstitution des dires qui élaborent leur ethos discursif, au sens de l'image construite en discours (Amossy, 1999), fournit l'occasion de découvrir ce moment particulier où la communication transfrontalière, exprimée depuis des positions discursives variées en Europe, passe d'un stade empirique et informel à un stade professionnel.

\section{Prolifération multilingue du discours eurorégional}

À partir du milieu des années quatre-vingt-dix, le discours eurorégional accompagne le déploiement et la visibilité des eurorégions sur le Web. Accessible à grande échelle, il prolifère en ligne depuis les années 2000, avant même la création du Règlement du GECT. Inscrit dans la lignée des corpus numériques qui nécessitent un outillage informatique pour le traitement, ce discours ouvre de vastes possibilités de réflexivité, de parcours et de mise en relation d'un contenu foisonnant et non linéaire (Rastier, 2011). Outre cette dimension numérique, le champ discursif eurorégional ne s'arrête pas aux frontières des langues pour garantir sa diffusion au sein d'espaces transfrontaliers. Sa visibilité est avérée dans plusieurs langues et il nécessite d'être réuni en corpus multilingue pour offrir une meilleure représentativité (Scholz, 2010). Enfin, la pluralité des contextes de production est à prendre en compte. Issu des mondes institutionnel, économique et médiatique, le corpus a été constitué selon la méthode du crawling thématique (Yapomo, 2013), qui consiste à identifier séparément dans chaque langue des terminologies pertinentes par rapport au thème eurorégional. Les multiples constituants discursifs ainsi réunis autour du « foyer thématique » eurorégional permettent, comme le recommande Maingueneau (2012), de construire des sous-corpus homogènes pour l'analyse.

Pour cet article, nous avons restreint l'observation à des énoncés traduisant la position institutionnelle eurorégionale parce qu'ils s'imprègnent d'un «degré de légitimité » et d'une «image préalable » constitutive d'un ethos prédiscursif (Amossy, 1999, p. 147). Nous excluons donc provisoirement les autres énoncés produits par la multitude d'acteurs économiques et médiatiques qui décrivent, racontent ou jugent les eurorégions.

La première phase de la collecte a permis de réunir des énoncés en français et en allemand, langues jugées prioritaires en raison de la concentration histo-

12. Selon le site officiel de l'Union européenne, pour la période 2007-2013, la coopération territoriale mobilise 8,7 milliards d'euros sur les 347 dédiés aux instruments de la politique régionale européenne (deuxième poste budgétaire immédiatement après celui de la politique agricole commune). La programmation pour la période 2014-2020 prévoit un budget en hausse pour la coopération territoriale, soit 9,6 milliards d'euros. 
rique des eurorégions le long des frontières de pays ou régions francophones (France, Belgique romane, Luxembourg, Suisse romande, Vallée d'Aoste en Italie) et germanophones (Allemagne, Autriche, est de la Belgique, Luxembourg, Suisse alémanique, Tyrol du Sud en Italie). Ces deux langues offrent à elles seules la possibilité de s'intéresser à de nombreuses eurorégions et l'espoir d'alimenter la comparaison grâce à des voix venues de pays - comme la France et l'Allemagne - aux positions discursives par ailleurs contrastées sur la mise en œuvre de réformes européennes (Angermüller, Scholz, 2013).

Pour envisager plus largement des discours issus d'autres zones européennes où se sont développées de nombreuses eurorégions au cours des dernières années, une seconde phase de la collecte s'est orientée vers des discours institutionnels disponibles en anglais, en néerlandais, en italien et en espagnol. Figurent ainsi dans la sélection des discours eurorégionaux venus du Nord (Pays-Bas, Danemark, Suède, Norvège, pays baltes), du Nord-Ouest (Irlande, Grande-Bretagne), de l'Est (Pologne, République tchèque, Slovénie) et du Sud (Italie, Grèce, Espagne, Portugal) de l'Europe.

Cette démarche a permis d'améliorer progressivement la représentativité des espaces considérés et d'enrichir l'analyse d'une vision contrastive, dans le sillage des travaux inscrits dans une perspective comparative (von Münchow et Rakotonoelina, 2006). Les 196 textes (103 853 mots) ${ }^{13}$ ainsi réunis sont puisés dans 32 sites web officiels ${ }^{14}$ et illustrent le discours institutionnel du tiers des eurorégions recensées par le Conseil de l'Europe, exprimé dans six langues officielles de l'Union.

Nous interrogeons ce sous-corpus à l'aide d'un logiciel de textométrie ${ }^{15}$ en formulant à titre exploratoire des requêtes sur les mots-pivots et les cooccurrences. L'analyse morphosyntaxique automatique dépendant de la langue considérée, les résultats sont répartis dans six partitions monolingues avant d'être utilisés comme des guides ou des étapes vers l'analyse qualitative.

Nous avons identifié trois conditions de comparabilité, démarche indispensable avant d'entreprendre l'analyse de documents authentiques produits dans des langues différentes (Claudel et Treguer-Felten, 2006, p. 24). La comparabilité provient d'abord de la proximité des contextes de production. Se focaliser sur les sites web institutionnels permet de se concentrer sur les énoncés

13. Les 103853 mots de ce sous-corpus institutionnel représentent un cinquième de notre corpus eurorégional complet (environ 500000 mots, en additionnant les discours institutionnels, économiques et médiatiques dédiés aux eurorégions).

14. Le directeur de publication et le nom de domaine garantissent le caractère officiel des sites web institutionnels sélectionnés.

15. Nous utilisons la plateforme libre TXM, mise à disposition par l'équipe du projet ANR Textométrie de l'École nationale supérieure de Lyon, en y connectant le composant modulaire TreeTagger, mis à disposition par l'université de Stuttgart pour l'étiquetage morphosyntaxique multilingue. Nous utilisons aussi des programmes développés sur mesure en Perl pour accélérer les requêtes multilingues et automatiser les extractions des résultats issus de l'ensemble du corpus. 
formulés par les eurorégions et sur leur démarche de construction d'une identité discursive. Ensuite, la ligne éditoriale partagée par cette communauté eurorégionale, entendue au sens d' ' institution qui reçoit sa cohérence de ses pratiques discursives» (Beacco, 2004), permet de réunir des documents produits dans des langues et des lieux différents : il s'agit toujours de présenter et de promouvoir l'eurorégion à la manière de sites institutionnels, même si l'on ne peut garantir que les procédés discursifs soient identiques. Un repère utile au projet comparatif est enfin fourni par la similarité des genres discursifs présents sur les sites institutionnels des eurorégions, matérialisés par des textes courts et destinés à une certaine pérennité : l'éditorial, le message de bienvenue, la déclaration d’intentions et la brochure de présentation.

\section{Légitimation discursive de l'espace eurorégional}

La détection automatique des noms communs montre un intérêt récurrent du discours institutionnel eurorégional pour des termes évoquant des configurations spatiales. Ceux-ci se classent dans les occurrences les plus fréquentes ${ }^{16}$ :

Tableau 1. Occurrences les plus fréquentes dans le discours institutionnel eurorégional, classées par ordre décroissant (le rang 1 correspond au terme le plus fréquent)

\begin{tabular}{ccccccc}
\hline Rang & Français & Italien & Espagnol & Anglais & Allemand & Néerlandais \\
\hline 1 & région & regione & - & region & - & - \\
\hline 2 & - & territorio & - & - & Euroregion & - \\
\hline 3 & - & - & eurorregión & - & - & - \\
\hline 4 & - & euroregione & región & - & Region & euregio \\
\hline 5 & eurorégion & - & territorio & area & - & - \\
\hline 6 & - & provincia & - & border & Grenzregion & regio \\
\hline 7 & territoire & - & - & - & - & provincie \\
\hline 8 & - & - & - & - & - & - \\
\hline 9 & espace & - & - & - & Europaregion & - \\
\hline
\end{tabular}

Le cotexte montre ensuite que la notion d'espace transfrontalier est directement reliée dans les énoncés à la stratégie d'avenir européenne et au projet de création de structures transfrontalières spécifiques défendu par la Commission européenne. Présentée comme un réservoir de solutions concrètes, l'eurorégion se passe de la référence à l'espace national pour s'affirmer, comme en atteste l'absence des mots «nation » ou «pays» dans le tableau. En examinant

16. Les cases vides du tableau font référence à des occurrences qui n’entrent pas dans le champ lexical de la configuration spatiale (par exemple président, projet, objectif). Ces occurrences ne sont pas mentionnées ici pour faciliter la lecture. 
plus en détail les positions énonciatives et l'environnement lexical, plusieurs similitudes avec la démarche déjà mise en œuvre parl'Union européenne pour susciter l'adhésion à son discours (Gobin, Cussó, 2002) peuvent être décelées. L'ancrage du discours institutionnel dans la référence à la figure d'autorité, la neutralisation de tout caractère polémique et une forte convergence lexicale se conjuguent pour proposer une vision consensuelle de l'espace eurorégional. Nous détaillons ci-dessous ces trois composantes à l'œuvre dans le discours.

\section{Référence à la figure d'autorité}

Le « contrat de communication» (Charaudeau et Maingueneau, 2002) lie le discours à un public idéal composé principalement d'habitants frontaliers (surtout les jeunes, cible récurrente et privilégiée), de partenaires institutionnels et économiques implantés le long des frontières, et de visiteurs occasionnels à titre personnel ou professionnel. Sa validité provient d'abord de l'information préalable fournie sur la capacité d'expertise des décideurs eurorégionaux (présidents, administrateurs, représentants officiels). Souvent issus du sérail politique régional, ceux-ci opèrent le transfert de leur capacité de pilotage vers un nouvel espace géographique, politique et économique. L'usage de la première personne et la référence à un tiers de prestige mettent en évidence une " autorité montrée» (Plantin, 1996) sous la forme de rappels d'un parcours professionnel ancré dans le territoire, de mentions d'un réseau relationnel stratégique ou d'affirmations de personnalités déterminées. Nous soulignons les éléments saillants dans deux exemples ci-dessous :

Nous avons des personnalités fortes : le Ministre belge de la Justice, Stefaan de Clerck, qui est aussi le Bourgmestre de Courtrai, Rudi Demotte, qui est le Ministreprésident de la Région wallonne, originaire de Tournai, moi-même, en tant que Vice-président, et puis Martine Aubry, présidente de Lille Métropole Communauté Urbaine. (Gilles Pargneaux, vice-président de l'Eurométropole Lille-Kortrijk-Tournai)

La position exceptionnelle du ministre de la Justice Poppenhäger dans les instances européennes et leurs réseaux associés sont un soutien important.17 (Franck Rossner, administrateur de l'Euregio Egrensis)

Les adresses ponctuelles aux publics donnent ensuite des précisions sur les conditions d'exécution et les contraintes du contrat. Les délégués en charge de sa réalisation (rédacteurs des sites web eurorégionaux, opérationnels impliqués dans les projets eurorégionaux) s'engagent, au présent et futur

17. Dans cet article, les énoncés en langues étrangères (italien, espagnol, anglais, allemand, néerlandais) sont traduits par nos soins. Le texte original est donné en notes, sans préciser la langue. Ici : Die hervorragende Position von Justizminister Poppenhäger in den europäischen Gremien und die dazugehörigen Netzwerke sind eine wichtige Unterstützung. Damit übernimmt auch Thüringen einen entscheidenden Gestaltungsanteil. 
de l'indicatif, à répondre aux attentes présumées d'orientation (nous espérons que vous trouverez votre chemin sur notre site), d'informations (nous vous informerons sur le site web) ${ }^{18}$ et de résultats (nous présentons les premiers résultats ${ }^{19}$. Le partage d'informations fait office de garantie de réalisation du contrat tandis que les invitations fonctionnent comme des facteurs de cohésion entre les parties liées (nous encourageons tout le monde à visiter ces deux fabuleuses ressources de notre région) ${ }^{20}$. C'est l'action qui prime dans les formulations et l'enjeu consiste à permettre l'exercice de l'autorité tout en s'assurant du «consentement de l'instance citoyenne» (Charaudeau, 2005, p.14).

\section{Neutralisation du caractère polémique}

Si l'on observe, à d'autres endroits du corpus (dans les discours économiques et médiatiques), des tensions relatives à la construction de l'espace eurorégional, le versant institutionnel étudié ici ne présente pas cette dimension polémique. La domination d'adjectifs à caractère déterminatif (Dufour, 2012) donne un ton consensuel au discours et sert surtout à préciser le périmètre géographique ou administratif (européen, territorial, régional) et fonctionnel (politique, économique, scientifique) attribué à l'espace eurorégional ${ }^{21}$ :

Tableau 2. Adjectifs les plus fréquents dans le discours institutionnel eurorégional

\begin{tabular}{ccccccc}
\hline Rang & Français & Italien & Espagnol & Anglais & Allemand & Néerlandais \\
\hline 1 & européen & europeo & universitario & european & grenzüberschreitend & engelstalig \\
\hline $\mathbf{2}$ & territorial & economico & - & cross-border & europäisch & europees \\
\hline 3 & transfrontalière & comune & - & joint & - & - \\
\hline 4 & économique & transfrontaliero & europeo & cultural & deutsch & internationaal \\
\hline 5 & - & culturale & - & local & gemeinsam & duits \\
\hline 6 & politique & - & educativo & regional & regional & - \\
\hline 7 & - & regionale & académico & academic & - & - \\
\hline 8 & régional & italiano & científico & - & niederländisch & - \\
\hline 9 & métropolitain & - & económico & international & weit & cultureel \\
\hline 10 & social & instituzionale & jurídico & national & - & duitstalig \\
\hline
\end{tabular}

18. wir werden Sie aufder Webseite informieren

19. presentiamo i primi risultati

20. we would encourage everyone to visit these fabulous two resources in our region

21. Les cases vides font référence à des adjectifs à visée argumentative, par exemple nouveau, important, intensivo, good... 
L'association de la configuration eurorégionale à des chiffres (statistiques de travailleurs, de stagiaires et d'apprentis recensés dans les eurorégions) ou à des organismes chargés de les produire (offices statistiques, observatoires interrégionaux) consolide ensuite le caractère indiscutable du discours institutionnel. Associés à une vision positive de l'eurorégion, les chiffres deviennent des indicateurs adaptés qui soulignent l'intensité et la qualité des échanges au sein des eurorégions. Entretenus de longue date et prometteurs d'un avenir radieux, ces échanges sont un argument au service d'une eurorégion dynamique et ouverte. Le transfert de biens, de services et de salariés contribue à garantir la continuité de l'espace eurorégional, propice aux transactions : les échanges n'ont guère cessé, les échanges ont continué22 (Eurorégion Alpes Méditerranée); de telles initiatives d'échanges aboutiront aussi au développement et à l'amélioration de compétences 23 (Euroregion Baltic); échange d'expérience avec les précurseurs (Euroregion Neisse-Nisa-Nysa); une plateforme d'échanges d'information'24 (Euregio BENEGO); stimuler les entrepreneurs par l'échange d'expériences 25 (Scheldemond euroregion)...

L'argument récurrent de continuité de l'espace eurorégional provient aussi de l'évocation d'un passé commun qui suffit à constituer un socle solide et accueillant pour la nouvelle configuration eurorégionale. Les références à l'histoire - déjà observées dans d'autres analyses de discours où une entité régionale est exposée comme une " unité naturelle » résultant du passé (Costa, Bert, 2011) - présentent l'eurorégion comme l'évolution normale d'une situation antérieure harmonieuse : une histoire commune (Eurégion Meuse-Rhin), des populations qui ont tissé ensemble et sans interruption au fil des siècles leur histoire (Region Transmanche), réunies par une histoire commune 26 (Europaregion Tirol-Süd Tirol-Trentino.

La mobilisation d'une mémoire commune s'appuie en outre sur la valorisation uniforme d'un patrimoine ultra-millénaire (Eurorégion Alpes-Méditerranée), d'un héritage culturel commun 27 (Euregio Barents), d'un patrimoine paysager 28 (Scheldemond euroregion) ou d'un patrimoine culturel commun 29 (EURORegion Elbe-Labe). Cet appel au patrimoine partagé et localisé rappelle la notion de "patrimoine de proximité», dont Utard (2005) interroge à la fois la contradiction logique (préoccupation du bien commun versus défense d'intérêts particuliers) et la légitimité symbolique (le bien ne devient patrimoine que sur décision d'une instance habilitée à produire et à gérer du symbole).

22. gli scambi hanno continuato

23. such exchange initiatives shall also result in the development and improvement of competence

24. een platform voor informatie-uitwisseling

25. door de ervaringsuitwisseling ondernemers te stimuleren

26. vereint durch eine gemeinsame Geschichte

27. common cultural heritage

28. landschappelijk erfgoed

29. Pflege des gemeinsamen kulturellen Erbes 
Exprimée ici à partir de points de vue extérieurs ou supérieurs à ceux des régions constitutives des eurorégions, la présentation de décisions communes annule enfin toute trace de référence nationale et de rivalité entre les régions : la définition d'une vision commune aux cinq régions 30 (Eurorégion AlpesMéditerranée); une coordination politique entre les cinq régions ${ }^{11}$ (Eurorregión Pirineos Mediterráneo); si l'on regarde cette micro-région d'un point de vue international 32 (Euroregion EuroBalkans). Il s'agit là de fondre les espaces eurorégionaux dans une volonté unifiée et acceptée sans trace de négociation préalable.

\section{Convergences lexicales}

Tout en lui donnant l'apparence de l'évidence et une tournure résolument positive, l'usage d'un vocabulaire imprécis impose à son tour au discours eurorégional une neutralisation et lui fait courir le risque d'un nivellement. Là où l'on attendrait des descriptions spécifiques des différents espaces eurorégionaux pour esquisser leur identité, le discours présente au contraire des univers lexicaux très proches. Les espaces transfrontaliers, dont l'histoire, les caractéristiques, le degré de formalisation et les besoins promotionnels varient considérablement, semblent entrer dans le moule d'une communication indifférenciée. Le plus souvent, l'explicitation de la complexité propre à chaque espace eurorégional n'aboutit pas. Il devient alors délicat d'évaluer dans quelle mesure la configuration eurorégionale permettra d'appliquer la stratégie de résolution de problèmes mise en avant dans le discours. Des extraits liés à la pertinence, à la cohérence et la volonté de devenir une référence donnent une idée saisissante de cette régularité lexicale observable dans la communication d'eurorégions pourtant bien différentes :

30. la definizione di una visione comune alle cinque regioni

31. una coordinación política entre las cinco regiones

32. the future of cross-border relations in the region, if we look at this micro-region from an international point of view 
Tableau 3. Échantillons de segments répétés caractérisant la notion d'espace eurorégional

\begin{tabular}{|c|c|c|}
\hline Eurorégion émettrice & Langue & Segments répétés (traduction en français) \\
\hline $\begin{array}{l}\text { - Meuse-Rhin } \\
\text { - West-Vlaanderen-Flandre-Dun- } \\
\text { kerque-Côte d'Opale } \\
\text { - Grande Région Saar-Lor-Lux }\end{array}$ & français & $\begin{array}{l}\text { territoires pertinents } \\
\text { espaces de coopération cohérents } \\
\text { territoire de référence }\end{array}$ \\
\hline $\begin{array}{l}\text { - Alpi-Mediterraneo } \\
\text { - Insubrica }\end{array}$ & italien & $\begin{array}{l}\text { spazio unico (espace unique) } \\
\text { spazio coerente (espace cohérent) } \\
\text { spazi eccezionali (espaces exceptionnels) } \\
\text { punto di riferimento (point de référence) }\end{array}$ \\
\hline $\begin{array}{l}\text { - Pirineos-Mediterráneo } \\
\text { - Alentejo-Algarve-Andalucía }\end{array}$ & espagnol & $\begin{array}{l}\text { espacio privilegiado (espace privilégié) } \\
\text { espacio atractivo (espace attractif) } \\
\text { espacios protegidos (espaces protégés) } \\
\text { espacio complejo (espace complexe) }\end{array}$ \\
\hline $\begin{array}{l}\text { - Baltic } \\
\text { - Barents } \\
\text { - East Border Region }\end{array}$ & anglais & $\begin{array}{l}\text { attractive space (espace attractif) } \\
\text { protected area (aire protégée) } \\
\text { coherent area (aire cohérente) } \\
\text { priority area (aire prioritaire) }\end{array}$ \\
\hline $\begin{array}{l}\text { - Salzburg-Berchtesgadener Land- } \\
\text { Traunstein } \\
\text { - Niederbayern-Oberösterreich- } \\
\text { Südbohmen }\end{array}$ & allemand & $\begin{array}{l}\text { attraktiven Lebensraum } \\
\text { (espace de vie attractif) } \\
\text { attraktiver Wirtschaftsraum } \\
\text { (espace économique attractif) }\end{array}$ \\
\hline - Euregio Scheldemond & néerlandais & $\begin{array}{l}\text { ruimtelijk-functioneel gebied } \\
\text { (zone spatio-fonctionnelle)... }\end{array}$ \\
\hline
\end{tabular}

L'adoption de la référence spatiale eurorégionale passe aussi par l'installation ponctuelle de la région métropolitaine transfrontalière polycentrique, du développement spatial polycentrique ou de l'agglomération polycentrique 33 (Grande Région). Ces segments, bien que peu nombreux, nous alertent sur l'importance que pourrait prendre, dans d'autres parties du corpus eurorégional, une rhétorique de la métropolisation et du polycentrisme, deux termes qui traduisent bien la notion d'intervention volontaire sur un espace classiquement connu (Allain, 2005).

La revue des emplois discursifs de la configuration urbaine permet en effet de déceler la participation ponctuelle de la ville et de la métropole à la légitimation de l'espace eurorégional. D'abord citée comme point de repère géographique pour localiser l'eurorégion (à proximité des grandes capitales 34 , Aix-laChapelle est la grande ville la plus occidentale) 35 , la ville devient un argument promotionnel important pourvaloriser l'espace eurorégional dès qu'une activité lui est associée. Lieu d'attractivité touristique au sein de l'espace eurorégional (cela encourage les habitants des deux villes à saisir l'occasion de

33. polyzentrischen Verdichtungsraum

34. proximity to major capital cities (Region Transmanche)

35. Aachen ist die westlichste Grosstadt (Grande Région) 
visiter l'autre ville) ${ }^{36}$ et, plus largement, lieu d'échanges et de socialisation (invités des villes «amies»)37, elle présente l'avantage de constituer une référence familière pour le public. Les autres emplois de la ville, dans le contexte de l'implantation de sièges ([l'Eurorégion] ayant son siège à la Maison de la Grande Région à Luxembourg-Ville) ou de la signature d'accords officiels (la Convention de coopération transfrontalière était signée dans la ville) ${ }^{38}$, contribuent également à l'institutionnalisation de l'espace eurorégional. Nous suggérons dès lors, en empruntant les deux concepts à Maingueneau (1999, p.79), de faire de la configuration urbaine une «scène » susceptible de faciliter «l'incorporation » imaginaire du public à la communauté de ceux qui adhèrent au discours eurorégional.

L'enjeu concerne en effet l'adoption d'un point de référence autre que celui auquel nous sommes habitués (la capitale au sein d'un espace national, ou la capitale régionale au sein d'un espace infranational) et à partir duquel situer la proximité vantée dans le discours eurorégional (proximitéavec ses citoyens, proximité de régions densément peuplées, proximité entre les acteurs publics, proximité des bassins de vie). La récurrence de données chiffrées (54800o habitants, 126 habitants au kilomètre carrê) 39 ou de positions de classement des centres urbains (Turin est la quatrième ville avec environ 900000 habitants) 40 montre aussi qu'un seuil démographique peut valoriser l'accessibilité et encourager la mobilité (principalement professionnelle et étudiante) en eurorégion. Du point de vue interdiscursif, cette préoccupation pour la mobilité évoque une filiation avec le Traité de Rome41 qui consacre dès 1957 la libre circulation des personnes à des fins essentiellement professionnelles. Du point de vue de la communication, les pratiques de médiatisation adossées à des classements et à l'argument de la mobilité sont déjà observées dans le discours des collectivités territoriales (Cardy, 2011) et des universités (Angermüller, Scholz, 2013; Gaspard, 2013) soucieuses de valoriser leurs performances.

Pour favoriser l'ancrage du repère eurorégional voulu par la politique européenne, le discours utilise des tournures inchoatives qui illustrent la création d'espaces nouveaux (l'organisation des villes en réseau est encore à ses débuts, [l'Europe élargie] construit des espaces de coopération interrégionaux). Des verbes de mouvement traduisent aussi l'ouverture d'anciens espaces clos (ouvre vers l'extérieur, ouvre à $360^{\circ}$ sur un territoire multiculturel), leur trans-

36. it encourages people from both towns's to take the opportunity to visit one another (East Border Region)

37. ospiti delle città « amiche » (Euroregione Adriatico-Ionica)

38. se firmaba en la ciudad el Convenio de cooperación transfronteriza (Eurorregión AlentejoAlgarve-Andalucía)

39. 548000 inhabitants, 126 inhabitants persq. $k m$ (Euregio Helsinki-Tallinn)

40. Torino è la quarta città con 900 ooo abitanti circa (Euroregione Alpi-Mediterraneo)

41. Le texte du Traité de Rome est disponible à l'adresse : http://www.constitutioneu.eu/cariboost files/trait_c3_a9_2ode_2orome.pdf 
formation (devenir un territoire d'interdépendance transfrontalier, suivre les évolutions des territoires européens) ou leur suppression (effacer les frontières, éliminer la frontière). Ces remous confortent la perspective déjà notée d'un glissement des centres névralgiques en Europe (Alliès, 2011), qui s'opère au détriment d'espaces classiquement délimités par les frontières nationales ou régionales et dont la destination est encore imprécise.

Pour conclure, nous rappelons que le discours institutionnel émis par les eurorégions s'inscrit dans une politique de coopération transfrontalière née après la seconde guerre mondiale. Il cherche à établir la pertinence des zones transfrontalières auprès de publics divers (habitants, jeunes, partenaires, visiteurs) et annonce une reconfiguration profonde de l'espace européen planifiée par les institutions européennes. Le sous-corpus multilingue étudié ici, matérialisé par des sites web institutionnels, reflète le positionnement du tiers des eurorégions recensées par le Conseil de l'Europe. L'observation des fréquences lexicales et l'analyse qualitative des énoncés dédiés à la notion d'espace ont permis de dégager trois régularités discursives : des manifestations d'autorité (autorité montrée, adresses ponctuelles aux publics), un effacement de la polémique (adjectifs déterminatifs, vision pacifique du passé) et une nette convergence lexicale indépendante du lieu d'émission du discours (équivalents multilingues de segments répétés). En vue d'incorporer le coénonciateur aux communautés émergentes que sont les eurorégions, le discours mobilise l'attention par l'évocation d'expériences (les échanges) et de références (les villes) communes. Par son insistance sur les échanges continus et une vision unifiée de l'histoire, il aplanit les différences entre eurorégions et procède à l'effacement d'une situation antérieure souvent marquée par des conflits transfrontaliers. L'hypothèse de départ selon laquelle le discours institutionnel des eurorégions tend à s'autolégitimer en gommant les résistances idéologiques nationales s'en trouve confirmée. Cet effacement des résistances nationales ne passe pas, comme nous en avions l'intuition, par l'invention d'identités eurorégionales spécifiques mais au contraire par une uniformisation des représentations qui rend les descriptions des eurorégions presque interchangeables. La légitimité institutionnelle des eurorégions ainsi forgée, conformément au programme de politique européenne, ouvre la voie à une image à la fois naturelle et idéalisée des zones transfrontalières.

\section{Références}

Allain Rémy, 2005, «Ville et proximité. Le point de vue d’un géographe-urbaniste», Mots. Les langages du politique, $\mathrm{n}^{\circ} 77$, Proximité, p. 128-136.

Alliès Paul, 2011, "La notion d'Eurorégion et sa mise en œuvre dans l'Union 
européenne», C. E. Pachedo Amaral éd., Autonomie régionale et relations internationales. Nouvelles dimensions de la gouvernance multilatérale, Paris, L'Harmattan, p. 245-255.

Amossy Ruth, 1999, Images de soi dans le discours. La construction de l'ethos, Lausanne, Delachaux et Niestlé.

ANGERMüLLER Johannes, Scholz Ronny, 2013, «Au nom de Bologne? Une analyse comparative des discours politiques sur les réformes universitaires en Allemagne et en France », Mots. Les langages du politique, $\mathrm{n}^{\circ} 102$, Les discours sur l'enseignement supérieur et la recherche, p. 20-36.

BEACCO Jean-Claude, 2004, "Trois perspectives linguistiques sur la notion de genre discursif », Langages, vol. XXXVIII, nº 153, p. 109-119.

CARDY Hélène, 2011, «Le discours identitaire dans les politiques de communication territoriale. La place de leur palmarès et de leur médiatisation », Mots. Les langages du politique, nº 97, Les collectivités territoriales en quête d'identité, p. 59-74.

Charaudeau Patrick, 2005, Les masques du pouvoir, Paris, Vuibert.

Charaudeau Patrick, Maingueneau Dominique, 2002, Dictionnaire d'analyse du discours, Paris, Le Seuil, p. 138-141.

Claudel Chantal, TReguer-Felten Geneviève, 2006, "Rendre compte d'analyses comparatives sur des corpus issus de langues/cultures éloignées», Les Cahiers du Cediscor, nº 9, Discours, cultures, comparaisons, P. von Münchow, F. Rakotonoelina éd., Paris, Presses de la Sorbonne nouvelle, p. 23-37.

Cole Alistair, PALmer Rosanne, 2009, "Logiques de territorialité et de régionalisation en Europe de l'Ouest », Revue d'études comparatives Est-Ouest, vol. XXXIX, p. 19-36.

COSTA James, BERT Michel, 2011, «De l'un et du divers. La région Rhône-Alpes et la mise en récit de ses langues», Mots. Les langages du politique, n 97 , Les collectivités territoriales en quête d'identité, p. 45-57.

Dufour Françoise, 2012, «Développement durable, humain. La cohérence discursive des contradictions", Mots. Les langages du politique, n 96 , Les discours politiques. Approches interactionnistes et multimodales, p. 81-96.

GASPARD Jeoffrey, 2013, «Le discours promotionnel des universités européennes. Homogénéité dans la compétitivité?», Mots. Les langages du politique, n0102, Les discours surl'enseignement supérieur et la recherche, p. 52-66.

Gobin Corinne, Cussó Roser, 2008, "Du discours politique au discours expert. Le changement politique mis hors débat?», Mots. Les langages du politique, n88, Discours politique, discours expert, p. 5-11.

GoEHRS Manuel, 2013, «Le groupement européen de coopération territoriale (GECT), outil contractuel de régulation territoriale », Jurisdoctoria, nº 9, p. 85-109.

Maingueneau Dominique, 2012, "Que cherchent les analystes du discours? ", Argumentation et analyse du discours, no9, p.2-14. En ligne : http://aad.revues. org/1354 (consulté le 12 mars 2014).

- 1999, "Ethos, scénographie, incorporation », R. Amossy éd., Images de soi dans le discours. La construction de l'ethos, Lausanne, Delachaux et Niestlé, p. 75-100.

MARIN Anaïs, 2012, "Coopérer à travers les frontières orientales de l'UE. Le modèle eurorégional et ses limites», Regard sur l'Est, nº62, Frontières recomposées à 
l'Est, en ligne : http://www.regard-est.com/home/breve_contenu.php?id=1377 (consulté le 10 mai 2014).

PERRIN Thomas, 2013, Culture et eurorégions. La coopération culturelle entre régions européennes, Bruxelles, Université libre de Bruxelles.

— 2011, "L'institutionnalisation de la coopération transfrontalière en Europe », Ceriscope Frontières, en ligne : http://ceriscope.sciences-po.fr/content/part2/linstitutionnalisation-de-la-cooperation-transfrontaliere-en-europe?page=6 (consulté le 19 mai 2014).

Plantin Christian, 1996, L'argumentation, Paris, Le Seuil, p. 88-93.

RASTIER François, 2011, La mesure et le grain. Sémantique de corpus, Paris, Honoré Champion, p. 9-52.

ScHolz Ronny, 2010, «Traiter le multilinguisme dans les discours politiques. Possibilités et limites d'une analyse lexicométrique dans un corpus composé des textes de différentes langues », intervention au séminaire du 19 mars 2010, Université ParisEst Créteil Val de Marne (Ceditec).

UTARD Jean-Michel, 2005, «Du patrimoine au "proximoine” ou le présent d'une illusion », Mots. Les langages du politique, n077, Proximité, p. 137-144.

von Münchow Patricia, Rakotonoelina Florimond, 2006, Les Cahiers du Cediscor, $n^{\circ}$ 9, Discours, cultures, comparaisons, Paris, Presses de la Sorbonne nouvelle.

WASSEMBERG Birte, 2009, "Le voisinage de proximité : les eurorégions "géopolitiques" aux frontières externes de l'UE (1993-2009)», Matériaux pour l'histoire de notre temps 1, nº 97-98, p. 45-49.

YAPOMO Manuela, 2013, "Construction de corpus multilingues : état de l'art », Actes de Recital 2013 : XVe Rencontre des étudiants chercheurs en informatique pour le traitement automatique des langues, 17-20 juin 2013, Les Sables d'Olonne, en ligne : http://www.taln2013.org/actes/www/RECITAL-2013/actes/recital-2013-long-005. pdf (consulté le 13 avril 2014). 
